\title{
Prognostic Value of Liver and Spleen Stiffness in Patients with Fontan Associated Liver Disease (FALD): A Case Series with Histopathologic Comparison
}

\author{
Massimo A. Padalino ${ }^{1, *,+}$, Liliana Chemello ${ }^{2, *,+}$ (D) , Luisa Cavalletto ${ }^{2}\left(\mathbb{D}\right.$, Annalisa Angelini $^{3}(\mathbb{C}$ \\ and Marny Fedrigo ${ }^{3}{ }^{10}$ \\ 1 Pediatric and Congenital Cardiac Surgery Unit, Department of Cardiac, Thoracic and Vascular Sciences \\ and Public Health, University of Padova Medical School, 35128 Padova, Italy \\ 2 Internal Medicine and Hepatology Unit, Clinica Medica 5, Department of Medicine-DIMED, University of \\ Padova Medical School, 35128 Padova, Italy; luisa.cavalletto@unipd.it \\ 3 Cardiovascular Pathology Unit, Department of Cardiac, Thoracic and Vascular Sciences and Public Health, \\ University of Padova Medical School, 35128 Padova, Italy; annalisa.angelini@unipd.it (A.A.); \\ marny.fedrigo@aopd.veneto.it (M.F.) \\ * Correspondence: massimo.padalino@unipd.it (M.A.P.); liliana.chemello@unipd.it (L.C.); \\ Tel.: +39-049-8212427 (M.A.P.); +39-049-8212294 (L.C.) \\ + These authors contributed equally to this work.
}

check for updates

Citation: Padalino, M.A.; Chemello, L.; Cavalletto, L.; Angelini, A.; Fedrigo, M. Prognostic Value of Liver and Spleen

Stiffness in Patients with Fontan

Associated Liver Disease (FALD): A Case Series with Histopathologic Comparison. J. Cardiovasc. Dev. Dis. 2021, 8, 30 https://doi.org/10.3390/jcdd8030030

Academic Editor: Monique R. M. Jongbloed

Received: 15 February 2021

Accepted: 12 March 2021

Published: 16 March 2021

Publisher's Note: MDPI stays neutral with regard to jurisdictional claims in published maps and institutional affiliations.

Copyright: (c) 2021 by the authors. Licensee MDPI, Basel, Switzerland. This article is an open access article distributed under the terms and conditions of the Creative Commons Attribution (CC BY) license (https:// creativecommons.org/licenses/by/ $4.0 /)$.

\begin{abstract}
The Fontan operation is the current surgical procedure to treat single-ventricle congenital heart disease, by splitting the systemic and pulmonary circulations and thus permitting lifespan to adulthood for the majority of newborns. However, emerging data are showing that Fontan-associated liver disease (FALD) is an increasing related cause of morbidity and mortality in patients with the Fontan circuit. We described the clinical, laboratory, and transient elastography (TE) findings in a case series of adults with the Fontan circuit, and also correlated data with post-mortem histological features, aimed to define the prognostic value of TE in the staging of FALD. All patients presented signs of a long-standing Fontan failure, characterized by reoperation need, systemic ventricle dysfunction, and FALD stigmata (liver and spleen enlargement, portal vein and inferior vena cava dilation, and abnormal liver function tests). Liver and spleen stiffness (LS and SS) values were indicative of significant liver fibrosis/cirrhosis and the presence of suggestive portal hypertension (LS mean 35.9; range 27.3-44.7 kPa; SS mean 42.1, range 32.2-54.5 kPa). Post-mortem evaluations confirmed a gross hepatic architecture distortion in all cases. All patients died from severe complications related to liver dysfunction and bleeding. TE correlated well with pathological findings and FALD severity. We propose this validated and harmless technique to monitor liver fibrosis extension and portal hypertension over time in Fontan patients, and to identify the optimal timing for surgical reoperations or orthotopic-heart transplantation (OHT), avoiding a higher risk of morbidity and mortality in cases with severe FALD.
\end{abstract}

Keywords: FALD; Fontan failure; cirrhosis; transient elastography; liver stiffness; liver histology

\section{Introduction}

Congenital heart diseases (CHDs) are rare conditions, with a reported incidence of 2-8/1000 live births, which need surgical repair in early infancy. The Fontan operation is a widely used surgical procedure that consistently extends the lifespan of cases with single-ventricle (SV) anatomy [1]. The Fontan circuit delivers systemic venous blood directly to pulmonary arteries in cases with SV, separating the pulmonary and systemic circulations, suddenly improving oxygen saturation in the blood. However, this pseudocirculation increases the central venous pressure and decreases cardiac output, leading to several long-term complications such as protein-losing enteropathy (PLE), plastic bronchitis, arrhythmias, heart failure, and hepatic fibrosis [2]. 
In particular, long-term liver congestion causes the development of cirrhosis and related complications (ascites, spontaneous bacterial peritonitis, encephalopathy, hepatorenal syndrome, gastro-esophageal bleeding, and hepatocellular carcinoma (HCC)) that are increasing incidence of morbidity and mortality related to Fontan correction [3]. Even if liver injury appears to be multifactorial, because of different noxae (hypoxemia, ischemiareperfusion, thrombosis, infections, or drug-toxicity) during multiple surgeries, the Fontan circuit creates the condition of venous hepatic stasis, with central vein and sinusoid dilation, abnormal liver perfusion, and a slow-down of portal blood flow. These unexpected and often misrecognized changes, related to the Fontan circuit, have been recently defined as Fontan-associated liver disease (FALD) [4].

We report here the clinical events in four adult patients with SV and Fontan correction who underwent redo-surgery for a failing Fontan, and who died from liver dysfunction and bleeding. We compared the non-invasive transient elastography (TE) with the post-mortem pathological features in order to define its prognostic value.

\section{Materials and Methods}

We considered four consecutive patients (three males, aged 22, 23, and 27 years and one female 50 years old) with SV and Fontan-circuit who presented with a long clinical period characterized by reoperation need, systemic ventricle dysfunction, and the presence of FALD signs (i.e., liver and spleen enlargement, portal and inferior vena cava (IVC) dilation, and abnormal liver function tests), who died soon after a surgical procedure or heart transplantation from complications related to an underestimated chronic liver disease.

All patients underwent an abdominal US with a Doppler analysis to investigate the echostructure and echogenicity of the liver, spleen, and kidney parenchyma and to detect any signs of portal hypertension (i.e., measuring spleen and portal vein $\varnothing$ and flow velocity rate) or systemic venous hypertension (i.e., measuring $\varnothing$ of IVC and suprahepatic veins). The main echocardiographic parameters were also considered and calculated for the classification of the functional heart performances and NYHA stage (i.e., measuring telediastolic ventricular volume, stroke volume, cardiac output, ejection fraction, IVC $\varnothing$, and collapsibility). The liver and spleen stiffness (LS and SS) were obtained using a FibroScan ${ }^{\circledR}$ (Echosens, Paris, France) following a standardized procedure, using an M probe, reporting values in kiloPascal (kPa; range 1.5-75 and 6.0-100, respectively) and with an interquartile range under $30 \%$.

A complete autopsy was performed according to the standard protocol adopted in our centre and previously published both for post-transplant [5] or adult CHD [6] cases. Histopathology was part of the complete autopsy, and the liver samples were collected and stained with Haematoxylin-Eosin and Masson's Trichromic (Diapath S.p.A., Martinengo (BG), Italy) for evaluation both of the extracellular matrix and fibrous pattern. Histopathologic semiquantitative methods were applied according to the morphological study by Kendall TJ et al. [7], particularly for the description of fibrosis deposition, sinusoids dilation, and hepatocytes/bile ducts damage, using the Metavir score [8] for inflammatory activity grading and fibrosis staging, and specially the congestive hepatic fibrosis (CHF) score [9] for grading the congestive (related to heart acute and chronic failure) and fibrotic (reactive to chronic liver damage) components.

\section{Results}

The clinical characteristics and surgical corrections of the patients are detailed in Table 1. Three cases showed a left systemic ventricle, and had major defects such as tricuspid atresia (TA) or double-inlet left ventricle (DILV) with transposition of great arteries (TGA) and pulmonary artery (PA) stenosis or aorta coarctation, and the other patient had hypoplastic left heart syndrome (HLHS). Only one case received a lateraltunnel at 3 years of age, while the others were corrected with a cavopulmonary at 20 and 15 years, or an atriopulmonary connection at 16 years of age-this case also received a 
redo-Fontan at 41-years. All cases needed reoperation: one for a pseudo-aneurism resection, one for a LVAD implantation, one for an ascending aorta replacement, and the last case received an orthotopic-heart transplantation $(\mathrm{OHT})$.

Table 1. Clinical and laboratory characteristics at the pre-operative presentation of cases.

\begin{tabular}{|c|c|c|c|c|}
\hline Clinical Characteristics & Case-1 $\sigma^{7}$ & Case-2 $\sigma^{7}$ & Case-3 9 & Case- $4 \sigma^{7}$ \\
\hline Type of CHD & HLHS & $\mathrm{TA}+\mathrm{TGA}+\mathrm{CoA}$ & DILV + TGA + PA stenosis & TA + TGA Sub-PA stenosis \\
\hline Type of sistemic ventricle & righ & left & left & left \\
\hline Age at first Fontan, years & 20 & 25 & 16 & 3 \\
\hline Type of Fontan & TCPC & TCPC & Atrio pulmonary & Lateral-tunnel \\
\hline Time from Fontan, years & 2 & 2 & 34 & 20 \\
\hline Redo Fontan & no & no & $\mathrm{TCPC}+\mathrm{BDG}+$ Ablation & no \\
\hline Age at last reoperation & 22 & 27 & 50 & 23 \\
\hline Type of reoperation & $\begin{array}{l}\text { Pseudo- } \\
\text { aneurysm resection }\end{array}$ & $\begin{array}{l}\text { Fenestration closure + } \\
\text { LVAD implantation }\end{array}$ & $\mathrm{OHT}$ & $\begin{array}{c}\text { Sub-Ao stenosis resection }+ \\
\text { AVR + Asc.Aorta } \\
\text { replacement }\end{array}$ \\
\hline Pre-operative NYHA class & III & III & III & IV \\
\hline Ejection fraction $(\mathrm{EF})(<55 \%)$ & $47 \%$ & $37 \%$ & $38 \%$ & $36 \%$ \\
\hline Protein-losing enteropathy & no & no & yes & no \\
\hline Dialysis session (>3 mos) & yes & yes & no & no \\
\hline Pre-operative arrhythmias & no & no & yes & no \\
\hline Pre-operative cyanosis & yes & yes & no & no \\
\hline \multicolumn{5}{|c|}{ Pre-Operative Laboratory Parameters (Normal Values) } \\
\hline PLTS, $\times 10^{3}(150-450)$ & 200 & 148 & 148 & 142 \\
\hline Lymphopenia, mm3 (<1.1) & yes & yes & yes & yes \\
\hline PT (INR) (0.9-1.2) & 1.18 & 1.91 & 1.66 & 1.36 \\
\hline AST / ALT/GGT, IU/L (<45) & $48 / 27 / 82$ & $36 / 28 / 99$ & $26 / 38 / 66$ & $35 / 26 / 56$ \\
\hline Bilirubin, umol/L (1.7-17) & 30 & 23 & 28 & 38 \\
\hline Albumin, g/L (4.2-5) & 28 & 32 & 28 & 30 \\
\hline Creatinine, umol/L (45-82) & 130 & 115 & 105 & 90 \\
\hline eGFR, ml/min/sqm (>90) & 48 & 50 & 62 & 80 \\
\hline CPT/MELD-XI score & $\mathrm{B} 8 / 13$ & B9/12 & B9/11 & $\mathrm{B} 8 / 11$ \\
\hline \multicolumn{5}{|c|}{ Pre-Operative Clinical and Instrumental Data } \\
\hline Liver, enlarged and stiff & yes & yes & yes & yes \\
\hline Spleen $\varnothing$, cm (normal < 12) & 13 & 19 & 17 & 19 \\
\hline Portal vein $\varnothing, \mathrm{mm}(<12)$ & 12 & 15 & 14 & 15 \\
\hline IVC Ø, $\mathrm{mm}(<17)$ & 24 & 25 & 26 & 28 \\
\hline Ascites decompensation & yes & yes & yes & yes \\
\hline
\end{tabular}

HLHS-hypoplastic left heart syndrome; TA—-tricuspid atresia; TGA-transposition of great arteries; CoA-aortic coarctation; DILV_double-inlet left ventricle; Ao_-aorta; PA—pulmonary artery; AVR—aortic valve replacement; BDG_-bi-directional Glen; LVAD_-left ventricular assist device; OHT_-orthotopic-heart transplantation; TCPC — total cavopulmonary connection. Clinical and lab parameters in bold characters are abnormal.

All cases have suffered from severe depression of the SV pump (ejection fraction (EF) $<47 \%$; three cases NYHA class III and one IV), and showed failing Fontan signs (two with chronic cyanosis and impaired kidney function needing dialysis, and the remaining one with PLE and arrhythmia). All cases presented episodes of abdominal ascites and oedema. In the lab tests, all had only a slight abnormal increase of bilirubin and GGT, but only one of AST, and no one of ALT. Three out of four cases showed initial thrombocytopenia and all lymphopenia, and scored a CPT class B8 or B9. Upon medical examination, patients documented an enlargement of liver and spleen, and upon abdominal-US they showed 
dilation of IVC and, in three cases, of the portal vein as well. At post-mortem pathological examination, all cases showed a gross architectural distortion of the hepatic lobule (grade 3 or 4) with sinusoidal dilation (grade 2 or 3) and a CHF score [9] ranging from 2B to 4, respectively, with the Metavir [8] score being F3 in Case 1 but F4 in the remaining ones.

In Figure 1, all cases showed a marked distortion of liver architecture, with fibrotic bridges arising from centrilobular vein and involving sinusoidal spaces and portal tracts, so as to manifest the tipical condition of "reverse cirrhosis". In particular, Case 1, staged Metavir F3 with a CHF score 2B, showed a mild fibrosis of the centrilobular veins and periportal tract, and delicate less extensive fibrosis of the sinusoidal spaces. In Case 2, the centrilobular fibrosis appeared to be thicker, with evident fibrotic bridging septa among the central veins and with a consistent sinusoidal fibrosis deposition. In Cases 3 and 4, was documented a gross architectural distortion of the liver, with diffuse sinusoidal fibrosis, and large septa (both with a Metavir of F4 and CHF scores of 3 and 4, respectively; see Table 2). In Case 4, there was also severe cholestasis, resembling the so called "nutmeg pattern".
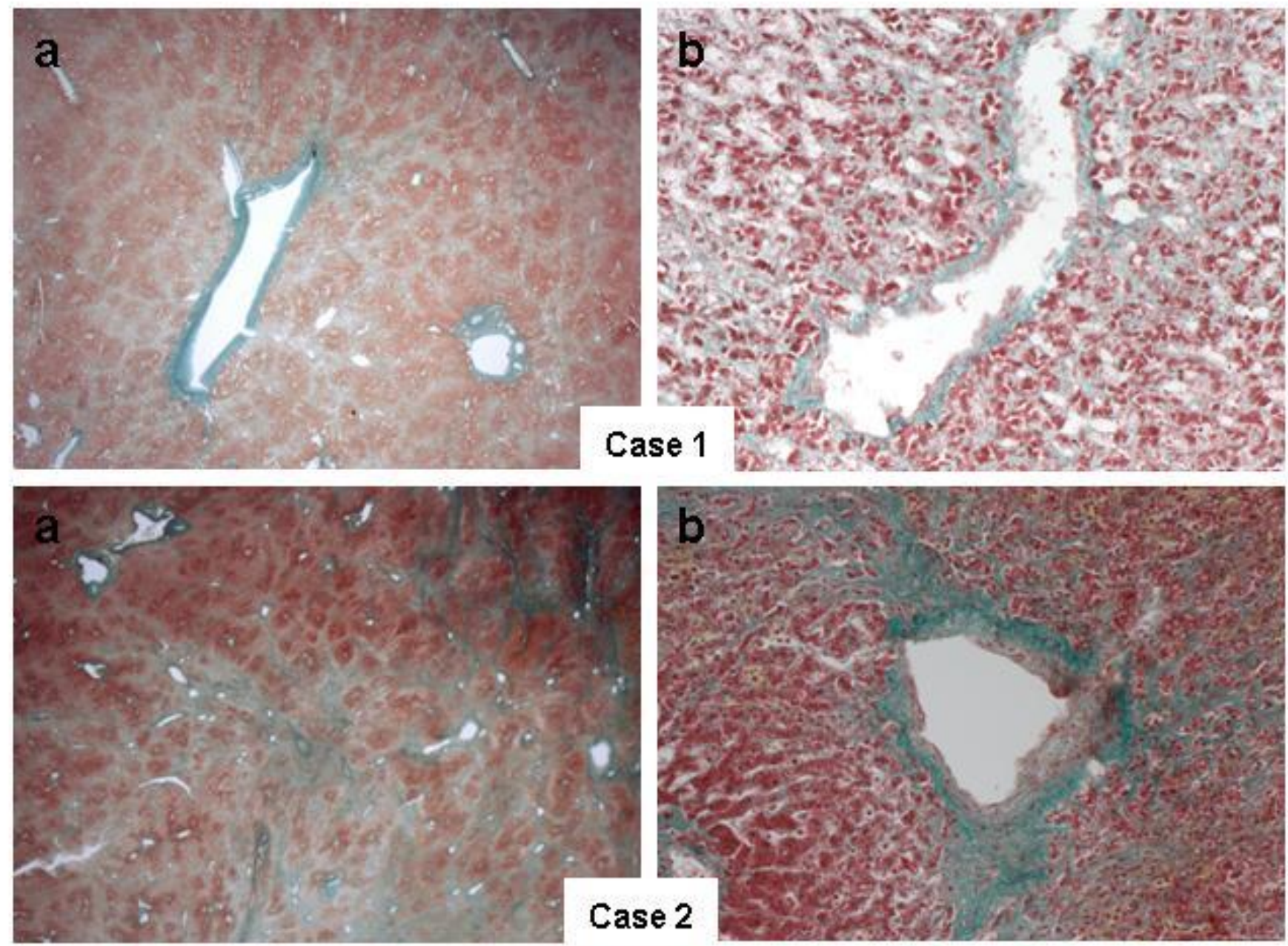

Figure 1. Cont. 

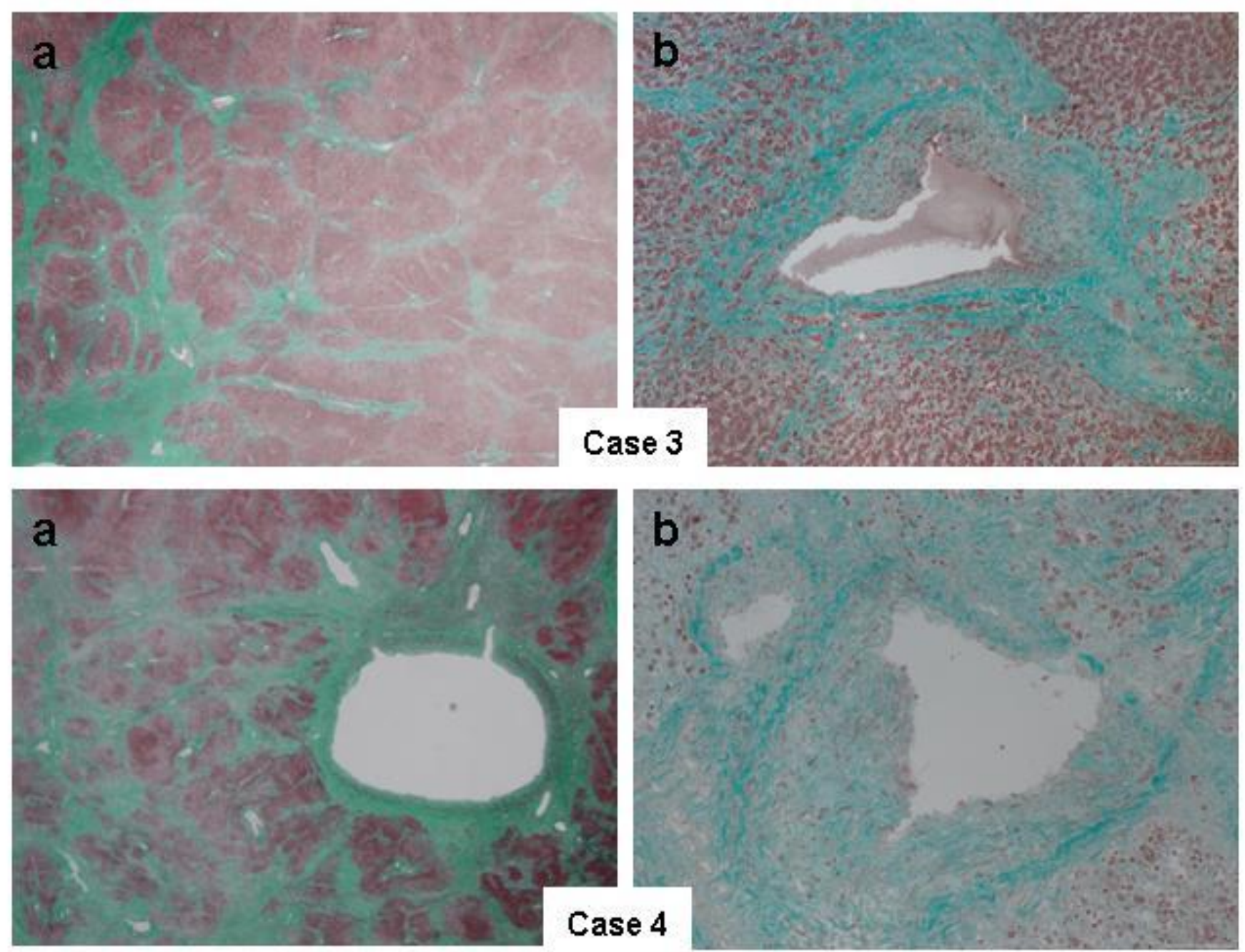

Figure 1. Liver histopathology obtained at autoptic examination by Masson's Trichromic staining: (a) a panoramic view $(\times 10$ magnification $)$ and $(\mathbf{b})$ close up view $(\times 40$ magnification $)$. In particular, the marked distortion of the liver structure, with fibrotic bridges arising from centrilobular veins and involving sinusoidal spaces and portal tracts, can be seen. Note the increasing thickness of fibrotic septa from Cases 1 to 4 , so as to manifest the characteristic condition of "reverse cirrhosis"; moreover, in Case 4, the presence of cholestasis also resembles the so called "nutmeg pattern".

Table 2 details the pathological features and the cause of death for the reported cases. Current and severe liver disease mainly contributed to the unfavourable fate and death in all patients. Very abnormal values of LS and SS (mean values of 35.9 and $46.9 \mathrm{kPa}$, respectively) were obtained by TE. Furthermore, LS correctly identified and graded the FALD, which was in accordance with the post-mortem pathological features, in order to fully satisfy its diagnostic and accurate role for all cases. In addition, SS was related to the presence of clinical significant portal hypertension and even bleeding risk at values over $50 \mathrm{kPa}$, as evidenced in Cases 2 and 3. 
Table 2. Description of the type and cause of death in relation to liver post-mortem pathological features and to transient elastography (TE) measurements.

\begin{tabular}{|c|c|c|c|c|}
\hline Patient & Case-1 $\sigma^{7}$ & Case-2 $\sigma^{7}$ & Case-3 + & Case-4 $\sigma^{7}$ \\
\hline Age at death, years & 22 & 27 & 50 & 23 \\
\hline Time of death from reoperation, days & 39 & 37 & 38 & 33 \\
\hline \multicolumn{5}{|c|}{ Type and Cause of Death } \\
\hline Clinical type of death & $\begin{array}{l}\text { Sudden respiratory } \\
\text { arrest, after sedation } \\
\text { for CVA positioning }\end{array}$ & $\begin{array}{c}\text { Acute on chronic liver } \\
\text { failure and } \\
\text { hemorragic shock }\end{array}$ & $\begin{array}{c}\text { Acute on chronic liver } \\
\text { failure and } \\
\text { hemorragic shock }\end{array}$ & $\begin{array}{c}\text { Acute on chronic liver } \\
\text { failure and intravasal } \\
\text { coagulation }\end{array}$ \\
\hline Pathological cause of death & $\begin{array}{l}\text { FALD with } \\
\text { multiorgan } \\
\text { deterioration }\end{array}$ & $\begin{array}{l}\text { Severe FALD with } \\
\text { multifocal bleeding } \\
\text { and multiorgan } \\
\text { damage }\end{array}$ & $\begin{array}{l}\text { Severe FALD with } \\
\text { enteric hemorrhage } \\
\text { and multiorgan } \\
\text { damage }\end{array}$ & $\begin{array}{l}\text { Severe FALD with } \\
\text { multiorgan } \\
\text { deterioration }\end{array}$ \\
\hline \multicolumn{5}{|c|}{ Histopathological Features [7] at Post Mortem Examination of Liver* } \\
\hline Gross architecture distortion (0-4) & 3 & 4 & 4 & 4 \\
\hline Sinusoidal fibrosis $(0-3)$ & 1 & 2 & 2 & 3 \\
\hline Sinusoidal dilation $(0-3)$ & 2 & 2 & 2 & 3 \\
\hline Bile duct reaction $(0-3)$ & 1 & 1 & 2 & 1 \\
\hline Cholestasis (0-2) & 1 & 1 & 1 & 2 \\
\hline Metavir score [8] & F3 & $\mathrm{F} 4$ & $\mathrm{~F} 4$ & $\mathrm{~F} 4$ \\
\hline Congestive hepatic fibrosis (CHF) score [9] & 2B & 3 & 3 & 4 \\
\hline \multicolumn{5}{|c|}{ Transient Elastography by Fibroscan } \\
\hline Liver stiffness, kPa (range 1.5-75 kPa) & 27.3 & 37.4 & 34.4 & 44.7 \\
\hline Spleen stiffness, kPa (range 6.0-100 kPa) & 30.6 & 56.4 & 52.2 & 48.7 \\
\hline
\end{tabular}

CVA - central venous access; * Hepatocellular damage in autoptic cases was not evaluable. All cases showed peri-sinusoidal and peri-venular fibrosis, but none showed iron deposition or inflammation.

\section{Discussion}

In this case series with SV-CHD and prolonged failing Fontan, the common finding of FALD represented the main comorbidity and the cause of death for all patients.

The prevalence of FALD is not well defined yet. Indeed, liver deterioration probably starts immediately after Fontan surgery [10], with the contribution of iatrogenic damage. However, this status often remains hidden for years, with the only evidence of liver venous congestion and stasis, revealing itself after many complications due to liver cirrhosis.

Post-Fontan liver histology has been reviewed in multiple small case series, including autopsy reviews and needle liver biopsies [3,11]. The histological analyses generally show lobular hepatic congestion and fibrotic distortion of the liver architecture in the majority of them. Although the liver biopsy is certainly a highly valuable diagnostic procedure, it is hardly applicable in the Fontan cohort, because of the high venous pressure and very common use of anticoagulation therapy-both conditions with a high risk of bleeding. In our cases, the histological features were performed according to various semiquantitative analyses [7-9]. In particular, Metavir [8] described a severe stage of liver cirrhosis in three out of four cases, and the CHF score [9] also confirmed a congestive pattern of grade 3 and 4 in these subjects (Cases 2-4).

On the other hand, we can rely in multiple non-invasive diagnostic imaging methods that can identify changes in the liver parenchyma (i.e., increased echogenicity, uneven texture, or liver surface nodularity), or can discover complications related to liver cirrhosis (i.e., liver and spleen enlargement, collateral circles, ascites, and hypervascular or HCC nodules) with sufficient specificity and accuracy [12]. The frequency of abnormal US findings has been shown to increase with time after the Fontan procedure [10], but contradictory correlations have been found between abnormal liver structures and biochemical parameters, hemodynamic data, age at Fontan, and other underlying diagnoses or ventricular morphologies. 
Here, the long duration of Fontan failure and its determining role on the increase of central venous pressure are certainly conditions that can be related to the entity of FALD in at least two cases (Cases 3 and 4). On the other hand, in the remaining two patients, where Fontan circulation was more recent (Cases 1 and 2), prolonged hypoxia may have played a main role in the progression of FALD. In fact, the hypoxic liver injury, also known as hypoxic hepatitis, has been described long time ago [13], caused by an inadequate oxygen uptake by the centrilobular hepatocytes, resulting in necrosis and residual fibrosis. This hypoxic insult can also affect nephrons and promote the development of ischemic acute tubular necrosis and kidney failure with the need for dialysis (as for Cases 1 and 2). Only one patient (Case 3) reported in the medical history a previous acute hepatitis $C$ with spontaneous viral clearance within 6 months from the acute phase of infection. Thus, the major pathogenetic mechanism of FALD in these cases can be considered to be heart pump failure, at preload and after load site, which induces both sinusoidal stasis and ischemia in major organs during the failing Fontan [2,4].

Recently, TE has been shown to be a promising novel non-invasive tool for long-term follow-up of cases with Fontan circuit [1,14]. In our case series, all patients had clinical evidences and TE data that were confirmatory of FALD. In addition, the evaluation by TE proved to be in agreement with the pathologic findings and this certainly strengthened its accurate role in staging liver fibrosis and portal hypertension. The LS showed elevated in all cases (mean of $35.9 \mathrm{kPa}$; range: $27.3-44.7 \mathrm{kPa}$ ), although common liver function tests (i.e., AST, GGT, and bilirubin) did not appear severely abnormal, and even the MELD-XI scores ranged low values as 11-13. However, LS is clearly related even to liver congestion, proven by IVC dilation in all cases, as well as to gross fibrotic lobular distortion, which develops over time. This is confirmed in the current literature, where a progressive LS increase in relation to a longer time from Fontan is described $[8,10]$.

Therefore, we believe, that TE still remains a practical and easy method to noninvasively distinguish between cases with and without progressive FALD. We found the LS follow-up to be a very useful and prognostic tool to monitor the related signs to Fontan circulation failing [15] and liver damage promotion in cases with Fontan circuit [16], particularly when found over the cut-off value of $22 \mathrm{kPa}$. Moreover, higher SS values appeared significantly related to the bleeding risk (as in Cases 2 and 3) [17]. Thus, we propose introducing TE for the evaluation of both congestion and fibrosis components of the liver and for the definition of portal hypertension, as part of the routine Fontan patients' clinical follow-up (see Figure 2), reserving a secondary role of the liver biopsy in this peculiar cohort. 


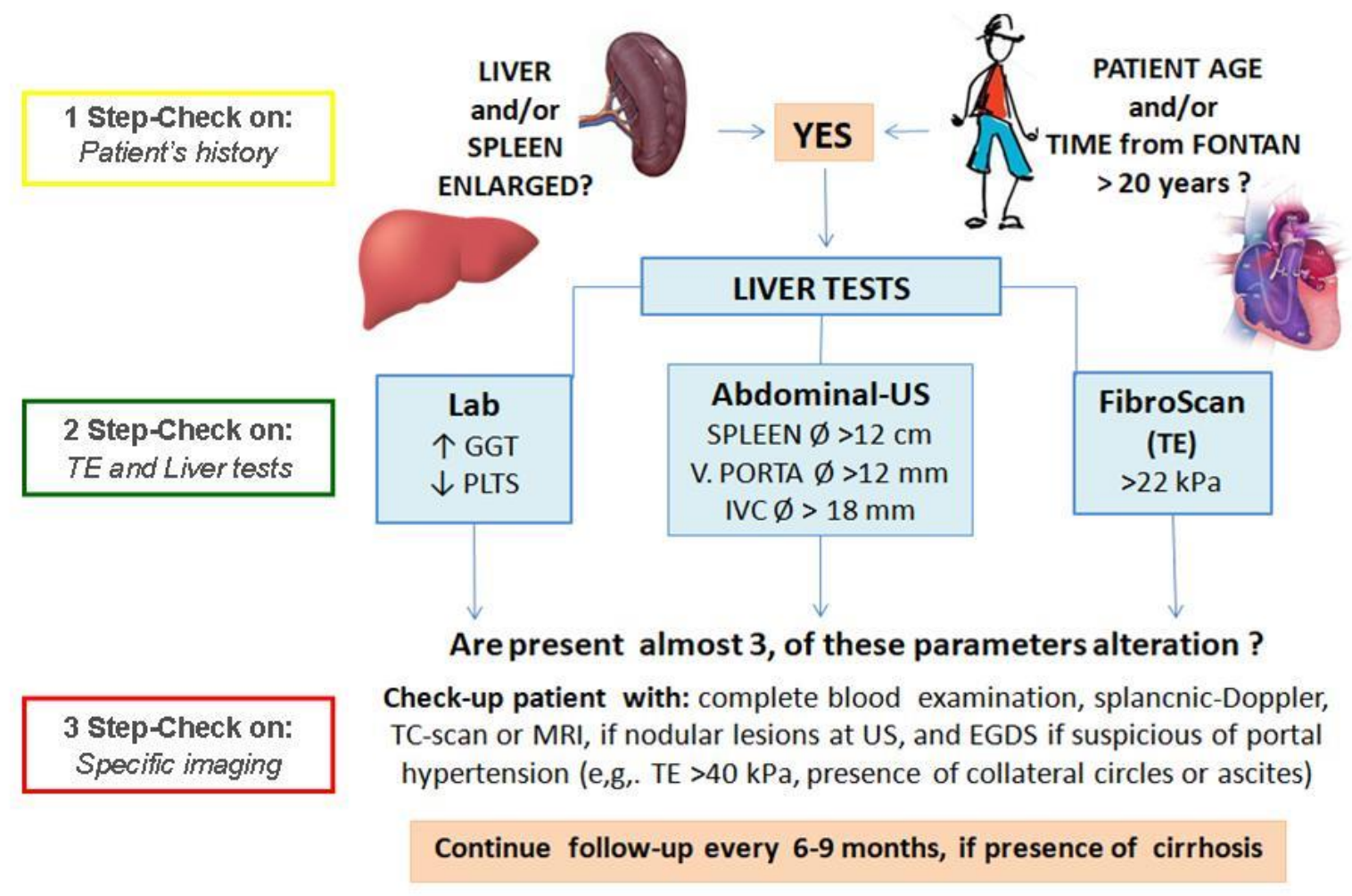

Figure 2. Step-by-step laboratory and instrumental approach to manage cases with signs or characteristics suggestive of Fontan-associated liver disease (FALD) in cases with single-ventricle (SV) and Fontan circuit.

\section{Conclusions}

FALD is a serious and life threatening condition during long-term outcomes after the Fontan operation, and its urgently needs an accurate evaluation as a combined heartliver transplantation may be required in selected cases. In our experience, TE has been proven to be a simple, accurate, and non-invasive method to monitor the stage of FALD through measurement of LS, but also to indicate Fontan circuit dysfunction early on. In addition, SS has revealed its diagnostic accuracy for the evaluation of portal hypertension and prediction of bleeding risk. Thus, we believe that all patients with major congenital heart disease and Fontan circuit should routinely undergo TE evaluation as part of their regular cardiologic follow-up in order to reveal hidden clinical conditions, especially liver related, and to possibly prevent postoperative complications that reduce patient survival.

Author Contributions: M.A.P. and L.C. (Liliana Chemello) shared first authorship, as both equally contributed to the conceptualization, data curation, formal analysis, investigation, methodology, supervision, validation; writing (original draft), and writing (review and editing). L.C. (Luisa Cavalletto) contributed to the data curation, methodology, supervision, validation, and writing (review and editing). A.A. contributed to the supervision, validation, and writing (review and editing). M.F. contributed to the data curation, methodology, supervision, validation, and writing (review and editing). All authors have read and agreed to the published version of the manuscript.

Funding: This research received no external funding.

Institutional Review Board Statement: This study was conducted according to the guidelines of the Declaration of Helsinki. Ethical review or approval was waived for this study because of the description of anonymous clinical cases, with no experimentation of drugs or procedures.

Informed Consent Statement: Patient consent was waived because of there was no active participation, as all were deceased. Authors kept the patients' identities anonymous.

Data Availability Statement: Not applicable. 
Acknowledgments: The Department of Medicine (DIMED) supported this paper with an internal grant fund by the Scientific Research project DOR 2019-1933494 of the University of Padova, Italy.

Conflicts of Interest: Authors declare no conflict of interest.

\section{References}

1. Téllez, L.; Rodrìguez-Santiago, E.; Albillos, A. Fontan-Associated Liver Disease: A Review. Ann. Hepatol. 2018, 17, 192-204. [CrossRef] [PubMed]

2. Fredenburg, T.B.; Johnson, T.R.; Cohen, M.D. The Fontan procedure: Anatomy, complications, and manifestations of failure. Radiographics 2011, 31, 453-463. [CrossRef] [PubMed]

3. Wu, F.M.; Kogon, B.; Earing, M.G.; Aboulhosn, J.A.; Broberg, C.S.; John, A.S.; Harmon, A.; Sainani, N.I.; Hill, A.J.; Odze, R.D.; et al. Liver health in adults with Fontan circulation: A multicenter cross-sectional study. J. Thorac. Cardiovasc. Surg. 2017, 153, 256-264. [CrossRef] [PubMed]

4. Daniels, C.J.; Bradley, E.A.; Landzberg, M.J.; Aboulhosn, J.; Beekman, R.H.; Book, W.; Gurviz, M.; John, A.; John, B.; Marelli, A.; et al. Fontan-associated liver disease: Proceeding from the American College of Cardiology Stakeholders Meeting, October 1-2, 2015, Washington DC. J. Am. Coll. Cardiol. 2017, 70, 3173-3194. [CrossRef] [PubMed]

5. Angelini, A.; Fedrigo, M.; Burke, M. The autopsy in Heart Transplantation. In The Pathology of Cardiac Transplantation a Clinical and Pathological Perspective; Leone, O., Angelini, A., Bruneval, P., Potena, L., Eds.; Springer: Berlin/Heidelberg, Germany, 2016; pp. 93-418. [CrossRef]

6. Angelini, A.; Di Gioia, C.; Doran, H.; Fedrigo, M.; Henriques de Gouveia, R.; Ho, S.Y.; di Gioia, C.; Fabre, A.; Gallagher, P.J.; Leone, O.; et al. Histopathologic features in congenital heart disease (CHD), from the Association for European Cardiovascular Pathology (AECVP). Virchows Arch. 2020, 476, 797-820. [CrossRef] [PubMed]

7. Kendall, T.J.; Stedman, B.; Hacking, N.; Haw, M.; Vettukattill, J.J.; Salmon, A.P.; Cope, R.; Sheron, N.; Millward-Sadler, H.; Veldtman, G.R.; et al. Hepatic fibrosis and cirrhosis in the Fontan circulation: A detailed morphological study. J. Clin. Pathol. 2008, 61, 504-508. [CrossRef] [PubMed]

8. Bedossa, P.; Poynard, T. An algorithm for the grading of activity in chronic hepatitis C. The METAVIR Cooperative Study Group. Hepatology 1996, 24, 289-293. [CrossRef] [PubMed]

9. Dai, D.F.; Swanson, P.E.; Krieger, E.V.; Liou, I.W.; Carithers, R.L.; Yeh, M.M. Congestive hepatic fibrosis score: A novel histologic assessment of clinical severity. Mod. Pathol. 2014, 27, 1552-1558. [CrossRef] [PubMed]

10. Deorsola, L.; Aidala, E.; Cascarano, M.T.; Valori, A.; Agnoletti, G.; Napoleone, C.P. Liver stiffness modifications shortly after total cavopulmonary connection. Interact. Cardiovasc. Thorac. Surg. 2016, 23, 513-518.

11. Goldberg, D.J.; Surrey, L.F.; Glatz, A.C.; Dodds, K.; O’Byrne, M.L.; Lin, H.C.; Fogel, M.; Rome, J.J.; Rand, E.B.; Russo, P.; et al. Hepatic fibrosis is universal following Fontan operation, and severity is associated with time from surgery: A liver biopsy and hemodynamic study. J. Am. Heart Assoc. 2017, 6, 179-185. [CrossRef] [PubMed]

12. Kim, T.H.; Yang, H.K.; Jang, H.J.; Yoo, S.J.; Khalili, K.; Kim, T.K. Abdominal imaging findings in adult patients with Fontan circulation. Insights Imaging 2018, 9, 357-367. [CrossRef] [PubMed]

13. Henrion, J.; Descamps, O.; Luwaert, R.; Schapira, M.; Parfonry, A.; Heller, F. Hypoxic hepatitis in patients with cardiac failure: Incidence in a coronary care unit and measurement of hepatic blood flow. J. Hepatol. 1994, 21, 696-703. [CrossRef]

14. Wu, F.M.; Opotowsky, A.R.; Raza, R.; Harney, S.; Ukomadu, C.; Landzberg, M.J.; Valente, A.M.; Breitbart, R.E.; Singh, M.N.; Gauvreau, K.; et al. Transient elastography may identify Fontan patients with unfavorable hemodynamics and advanced hepatic fibrosis. Congenit. Heart Dis. 2014, 9, 438-447. [CrossRef] [PubMed]

15. Greenway, S.C.; Crossland, D.S.; Hudson, M.; Martin, S.R.; Myers, R.P.; Prieur, T.; Hasan, A.; Kirk, R. Fontan-associated Liver Disease: Implications for Heart Transplantation. J. Heart Lung Transplant. 2016, 35, 26-33. [CrossRef] [PubMed]

16. Kutty, S.S.; Peng, Q.; Danford, D.A.; Fletcher, S.E.; Perry, D.; Talmon, G.A.; Scott, C.; Kugler, J.D.; Duncan, K.F.; Quiros-Tejeira, R.E.; et al. Increased hepatic stiffness as consequence of high hepatic afterload in the Fontan circulaton: A vascular Doppler and elastography study. Hepatology 2014, 59, 251-260. [CrossRef] [PubMed]

17. Giunta, M.A.; Conte, D.; Fraquelli, M. Role of spleen elastography in patients with chronic liver diseases. World J. Gastroenterol. 2016, 22, 7857-7867. [CrossRef] [PubMed] 